\title{
Tachycardia Following Ganglion Impar Block
}

\section{E. Ngugi Kinyungu, Troy Buck, Joseph R. Holtman}

Department of Anesthesiology, Division of Pain Management and Pain Medicine, Loyola University Medical Center, Maywood, USA.

Email:mukorak@yahoo.com

Received June $21^{\text {st }}, 2012$; revised July $25^{\text {th }}$, 2012; accepted September $14^{\text {th }}, 2012$

\begin{abstract}
Objective: To understand an unusual complication of a low risk procedure. Design: This article chronicles the side effect of a Ganglion Impar Block. Setting: Loyola University Medical Center Outpatient Chronic Pain Clinic. Patients: One. Results: Our patient had tachycardia after a Ganglion Impar Block. Conclusions: An unusual complication of a ganglion impar block can be increased heart rate.
\end{abstract}

Keywords: Ganglion Impar Block; Tachycardia

\section{Case Report}

The ganglion impar is solitary retroperitoneal structure located at the level of the sacro-coccygeal junction. Its blockade with local anesthetic is a technique used for the management of chronic perirectal pain, visceral pain in perineal area. Few complications have been reported for this procedure. We present here a case of tachycardia following a ganglion impar block. The patient consented for presentation of this case report.

A $67 \mathrm{y} / \mathrm{o} \mathrm{f}$ presented to the outpatient Chronic Pain Clinic for treatment of chronic perirectal pain. The patient had sustained a spinal cord injury at L1 in 1992 which left her paraplegic and wheelchair bound. Over time she developed perirectal pain. The patient was managed at the pain clinic for several years for this pain. Several medications were tried over time without resolution of the pain. Her pain continued and we subsequently discussed ganglion impar block with the patient.

The patient presented to the pain clinic for the procedure. The patient was under fasting guidelines of no solids by mouth for the procedure for 8 hours. She was able to drink clear liquids up to 2 hours prior to the procedure. Her pre procedure vital signs were a HR (heart rate) of 110, BP (blood pressure) 104/78, perirectal pain 8/10, weight of $80 \mathrm{lb}$, height 5'6”. She was consented for the ganglion Impar Block

In the fluoroscopy suite the patient was placed in the prone position and her back prepped and draped under sterile conditions. Using Anterior-Posterior (A/P) and lateral fluoroscopic guidance, the sacrococcygeal junction and tip of coccyx were identified. A trans-acrococcygeal approach to the ganglion impar was performed
[1]. Three cc of $1.5 \%$ lidocaine was used for skin infiltration using a 27 Ga needle. An 18 gauge 1.5 inch needle was then inserted through the sacrococcygeal ligament in the midline (Figure 1).

Needle position was confirmed under fluoroscopy and with 1 cc of contrast dye (Figures 2 and 3 ).

Five cc of $0.25 \%$ bupivacaine was then injected after negative aspiration for heme. The patient tolerated the procedure well in the fluoroscopy suite. She was then transferred to the recovery room in her wheel chair.

After 5 minutes in the recovery room the patient's heart rate was noted to be in the 150's, regular rhythm, BP 115/68. The patient was unclear as to whether her perirectal pain was improved. She indicated that she felt anxious. Patient was given one liter of $0.9 \%$ normal saline IVF (intravenous fluids) in the recovery room and it

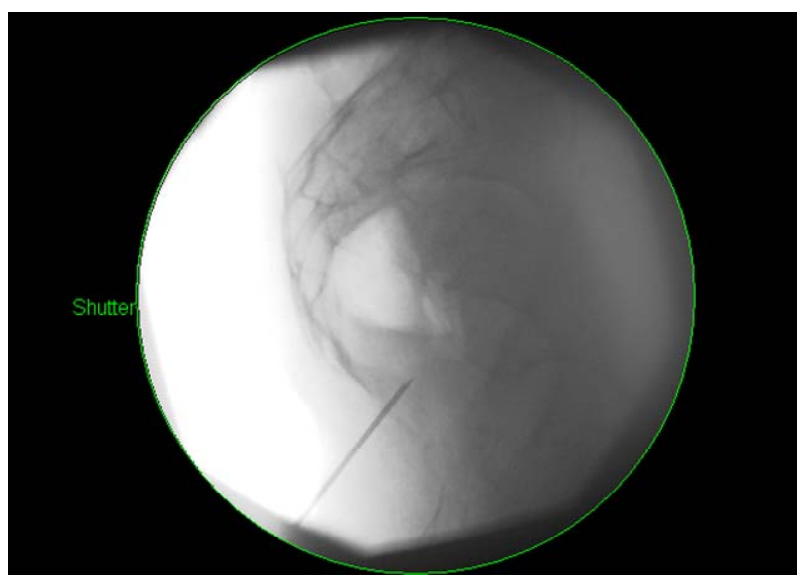

Figure 1. $18 \mathrm{Ga} 1.5$ inch needle through the sacrococcygeal junction. 


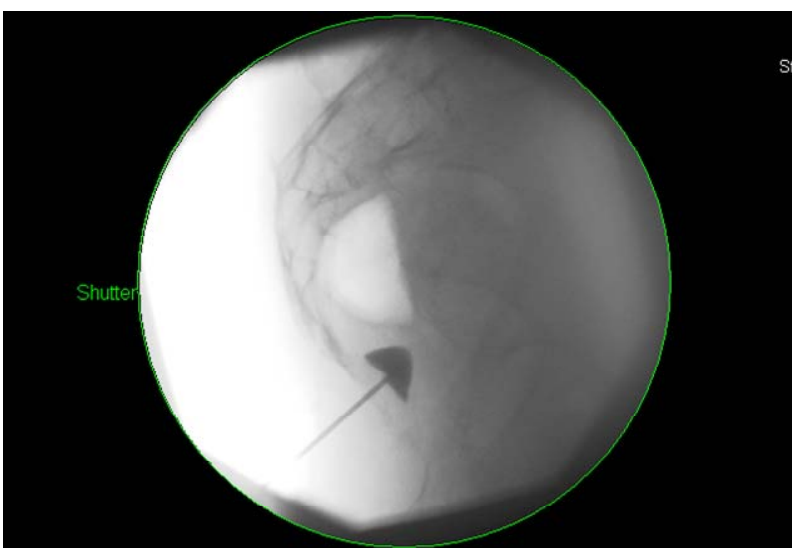

Figure 2. Injection of $1 \mathrm{ml}$ contrast in lateral view.

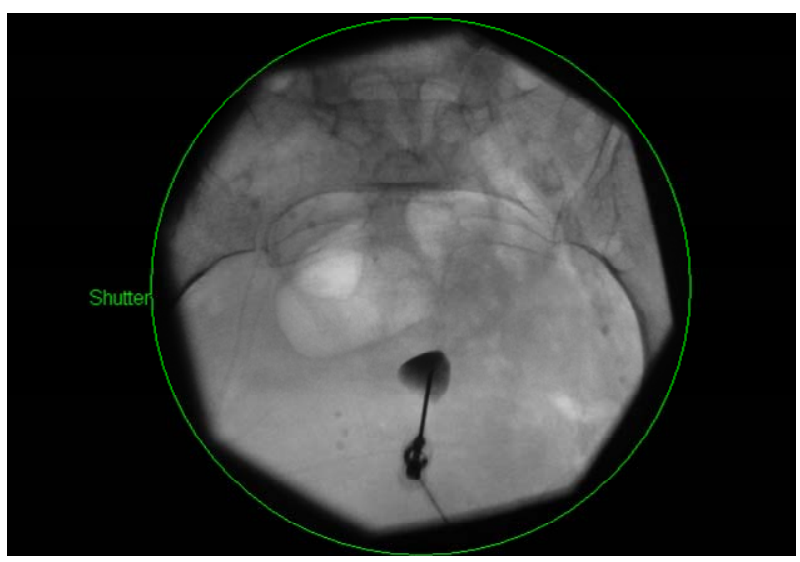

Figure 3. A-P view of contrast injection.

was noted that her HR decreased to the 130's. A decision was made to transfer the patient to the emergency room for further management.

In the emergency room the patient had a CBC (complete blood count), BMP (basic metabolic panel), urine culture and a UA (Urine Analysis) done. She also received another liter of IVF and $2 \mathrm{mg}$ of morphine for pain. Her tachycardia resolved after this liter of IVF (approximately one hour after the procedure) and her pain resolved significantly. She was released home after 4 hours in the emergency room-she stated she had no perirectal pain on discharge. We called the patient the next day at home to check on her and the patient stated she had zero perirectal pain.

\section{Ganglion Impar Blockade}

The Ganglion impar lies anterior to the sacrococcygeal junction. It is a termination of paired paravertebral sympathetic chains. Visceral afferents innervating perineum, distal rectum, anus, distal urethra, vulva and distal third of vagina converge at the ganglion impar [2]. Ganglion impar blockade has been used for chronic perineal pain from malignancies [3]. Potential complications for this block include puncture of the rectum, epidural spread of medication, bleeding or local trauma, and vasodilatation after sympathectomy.

\section{Discussion}

The etiology of the tachycardia after this block is unclear.

We can hypothesize a possible reason involving vasodilatation in a patient who was volume depleted to begin with. This patient has a history of poor PO intake and with this in the background plus a fasting period for this procedure it is possible that the effects of the ganglion impar block caused a decrease in preload with an increase in heart rate as compensation mechanism. This hypothesis is supported by the fact that the patient's tachycardia resolved with volume resuscitation.

The block was successful as evidenced by the fact that her pain resolved and up to 3 months later the patient still had good pain control. It may be prudent to ensure adequate hydration in patients prior to a ganglion impar block.

\section{REFERENCES}

[1] K. J. Wemm and L. Sabersky, "Modified Approach to Block the Ganglion Impar (Ganglion of Walther)," Regional Anesthesia, Vol. 20, No. 6, 1995, pp. 544-545.

[2] Oscar de Leon-Casacola, "Neurolysis of the Sympathetic Axis for Cancer Pain Management,” In: H. T. Benzon, J. Rathmell, C. Wu, D. Turk and C. Argoff, Eds., Raj's Practical Management of Pain, 4th Edition, Elsevier, Philadelphia, 2008, pp. 923-924.

[3] J. B. Swofford and D. M. Ratzman, "A Transarticular Approach to Blockade of the Ganglion Impar (Ganglion of Walther)," Regional Anesthesia and Pain Medicine, Vol. 23, Suppl. 3, 1998, p. 103. doi:10.1097/00115550-199823031-00103 